\title{
Optical technique for the automatic detection and measurement of surface defects on thin metallic wires
}

\author{
Luis Miguel Sanchez-Brea, Philip Siegmann, Maria Aurora Rebollo, and Eusebio Bernabeu
}

\begin{abstract}
In industrial applications of thin metallic wires it is important to characterize the surface defects of the wires. We present an optical technique for the automatic detection of surface defects on thin metallic wires (diameters, $50-2000 \mu \mathrm{m}$ ) that can be used in on-line systems for surface quality control. This technique is based on the intensity variations on the scattered cone generated when the wire is illuminated with a beam at oblique incidence. Our results are compared with those obtained by atomic-force microscopy and scanning-electron microscopy. (C) 2000 Optical Society of America
\end{abstract}

OCIS codes: $240.0240,120.6660,240.6700,240.5770,120.0120$.

\section{Introduction}

Thin metallic wires are produced for a great number of applications, such as for electronics, semiconductor manufacturing, automotive, xerography, and chemical and biomedical industries. For example, thin metallic wires can be found in electronic circuits, high-precision magnetic coils, air bags, high-pressure filters, etc. The systems that are customarily used for wire inspection measure the diameters of the wires. ${ }^{1-3}$ However, the surface quality of thin metallic wires plays an important role in many of these applications because defects on a wire's surface may change some physical and chemical properties of the wire. For this reason it is important to detect the wire surface defects to carry out effective quality control in the manufacturing process. As a result of the fabrication process of the wires ${ }^{4}$ the most common kinds of defect that appear on the wire surface are longitudinal defects such as scratches, drawing grooves, and protuberances, although there are other common defects such as holes, noses, flaking, and roughness zones. ${ }^{5}$ The characterization of rough-

L. M. Sanchez-Brea (optbrea@eucmos.sim.ucm.es), P. Siegmann, and E. Bernabeu are with the Departmento Optica, Universidad Complutense de Madrid, Facultad de Ciencias Físicas, Ciudad Universitaria, s/n. 28040 Madrid, Spain. M. A. Rebollo (mreboll@aleph.fi.uba.ar) is with the Laboratorio Aplicaciones Ópticas, Departamento Física, Universidad de Buenos Aires, Facultad de Ingeniería, Paseo Colón 850, 1063, Buenos Aires, Republic of Argentina.

Received 26 May 1999; revised manuscript received 7 October 1999.

0003-6935/00/040539-07\$15.00/0

(C) 2000 Optical Society of America ness and defects on plane surfaces has been studied extensively. ${ }^{6-9}$ However, many of these techniques are not accurate when they are used for wires. Thus new procedures need to be developed for this purpose. ${ }^{10-13}$ In this paper we propose an optical technique to detect and measure the defects that appear on the wire surface. This technique is based on the intensity variations produced by the defects when the wire is illuminated with a laser beam at oblique incidence. By analyzing these intensity variations one can obtain the widths and lengths of the defects. Where the interest is not actually in the location of the defects but in the measurement of the wire surface quality, as in on-line systems for wire surface control, a comprehensive analysis of the location of defects on the surfaces is not necessary. Thus we propose a parameter that can be used to determine the quality of the wire surface. This technique has been compared with laboratory test measurements of the wire surface by scanning-electron microscopy (SEM) and atomic-force microscopy (AFM).

\section{Geometrical Conical Diffraction}

When a light beam is incident obliquely upon a cylindrical surface with respect to the wire axis, the reflected light generates a cone. ${ }^{14,15}$ According to the geometrical theory of diffraction, ${ }^{16}$ the intensity distribution at the cone has two components ${ }^{17}$ : a diffractive one that is important only for angles close to the diffraction maximum and a geometrical one that can be explained by geometrical optics (see Fig. 1). Then, except for angles close to the diffraction maximum angle, one can use a geometrical model to 


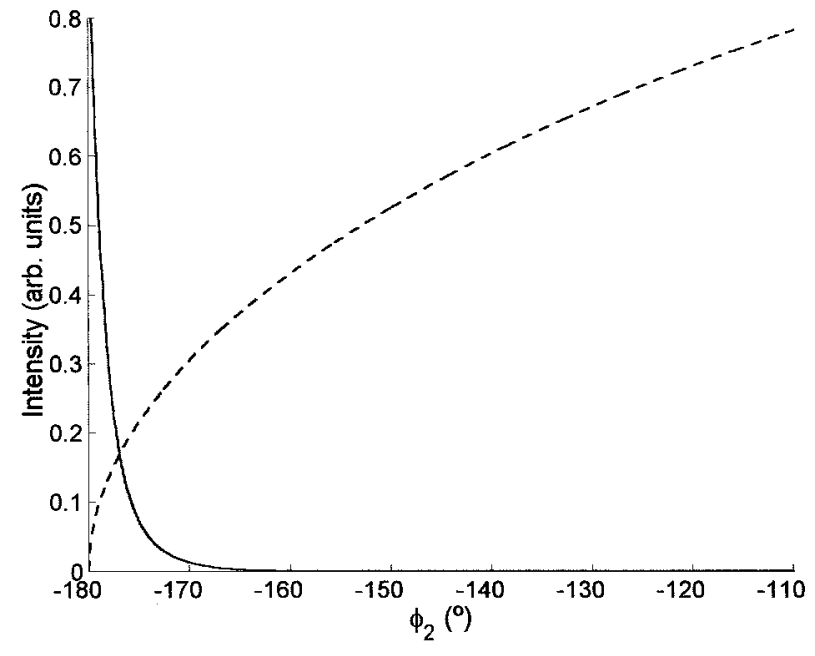

Fig. 1. Diffraction and geometrical contributions to the scattering pattern according to the geometrical theory of diffraction for a perfect, smooth cylindrical wire of diameter $300 \mu \mathrm{m}$ : solid curve, diffraction contribution; dashed curve, geometrical contribution. The wavelength is $\lambda=675 \mathrm{~nm}$, and the incidence is normal to the wire axis. $\phi_{2}$ is the angle with respect to the maximum of the geometrical contribution.

study the scattering of light by a metallic wire. Let us consider a uniform plane wave whose direction is

$$
\mathbf{k}_{1}=2 \pi / \lambda\left(\cos \theta_{1} \hat{z}-\sin \theta_{1} \hat{x}\right),
$$

where $\lambda$ is its wavelength, $\theta_{1}$ is the angle of the incident beam with respect to the wire axis, and $\hat{x}, \hat{y}$, and $\hat{z}$ represent unit vectors in the orthogonal directions. This beam is incident upon the wire surface, the normal of which is

$$
\hat{n}=\cos \phi_{1} \hat{x}+\sin \phi_{1} \hat{y},
$$

where $\phi_{1}$ is the angle between the incident beam and the normal to the wire surface, as shown in Fig. 2(a). According to geometrical optics, the output rays are specularly reflected. Each ray of the incident beam impinges upon the wire at a different angle $\phi_{1}$. To calculate the direction of the output rays we express Eq. (1) as

$$
\mathbf{k}_{1}=\mathbf{k}_{1_{\|}}+\mathbf{k}_{1 \perp}
$$

where $\mathbf{k}_{1}=\left(\mathbf{k}_{1} \cdot \hat{n}\right) \hat{n}$ is the projection of $\mathbf{k}_{1}$ upon the normal to the surface $\hat{n}$ and $\mathbf{k}_{1 \perp}=\mathbf{k}_{1}-\mathbf{k}_{1}$. The direction of the reflected rays can be calculated from $\mathbf{k}_{2}=\mathbf{k}_{2_{\|}}+\mathbf{k}_{2 \perp}$ [see Fig. 2(a)], where

$$
\begin{aligned}
\mathbf{k}_{2 \|} & =-\mathbf{k}_{1_{\|}}, \\
\mathbf{k}_{2 \perp} & =\mathbf{k}_{1 \perp} .
\end{aligned}
$$

Once $\mathbf{k}_{1_{\|}}$and $\mathbf{k}_{1 \perp}$ are obtained from Eqs. (1)-(3), $\mathbf{k}_{2}$ is

$$
\begin{aligned}
\mathbf{k}_{2}\left(\phi_{1}, \theta_{1}\right)= & k\left(\cos \theta_{1} \hat{z}+\sin \theta_{1} \cos 2 \phi_{1} \hat{x}\right. \\
& \left.+\sin \theta_{1} \sin 2 \phi_{1} \hat{y}\right) .
\end{aligned}
$$

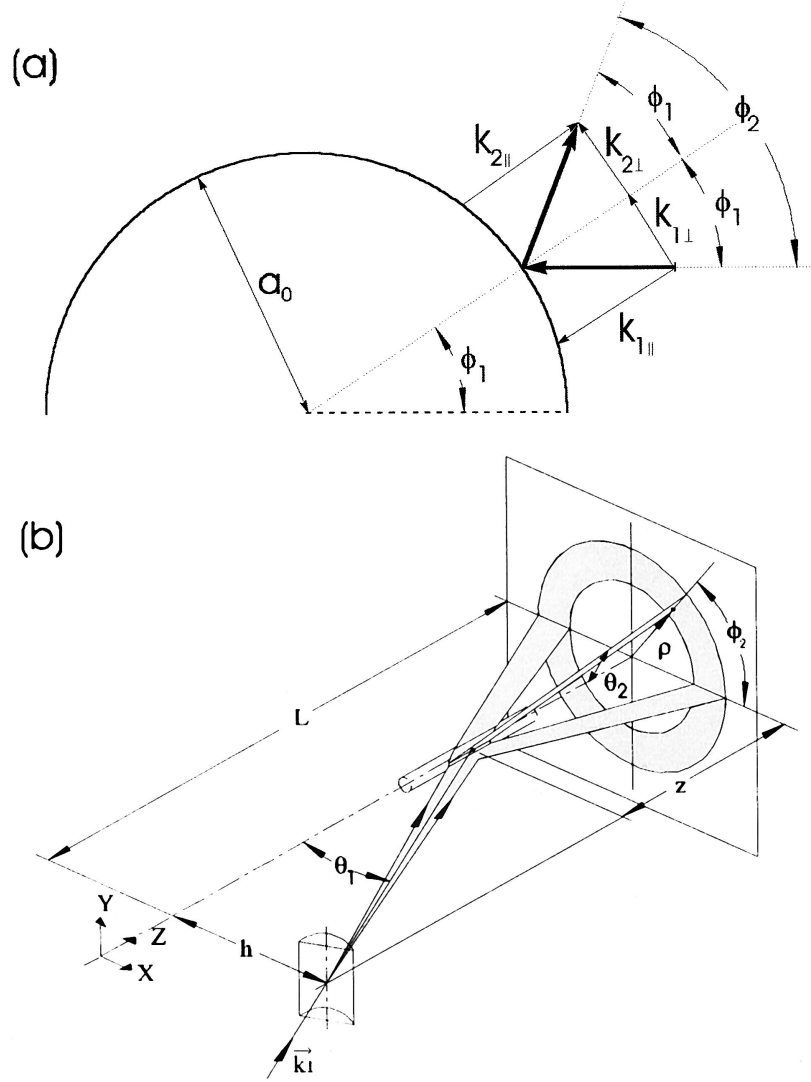

Fig. 2. (a) Transverse representation of the wire, showing the reflection of rays. (b) Sketch of the cone generated by the wire when the wire is illuminated with a laser beam at oblique incidence.

Comparing Eq. (5) with an arbitrary output ray in cylindrical coordinates, $\mathbf{k}_{2}\left(\phi_{2}, \theta_{2}\right)=k\left(\cos \theta_{2} \hat{z}+\sin \theta_{2}\right.$ $\left.\cos \phi_{2} \hat{x}+\sin \theta_{2} \sin \phi_{2} \hat{y}\right)$, we obtain

$$
\begin{aligned}
\theta_{2} & =\theta_{1}, \\
\phi_{2} & =2 \phi_{1},
\end{aligned}
$$

where the angles $\phi_{2}$ and $\theta_{2}$ are defined in Fig. 2(b). As a consequence, the scattered light generates a cone in the domain of directions. However, in real space the rays are shifted relative to one another, and the image obtained at a screen perpendicular to the wire axis is not exactly a cone but is given by ${ }^{14}$

$$
\phi_{2}=\tan ^{-1}\left(\frac{a_{0} \sin \phi_{1}+\rho \sin 2 \phi_{1}}{a_{0} \cos \phi_{1}+\rho \cos 2 \phi_{1}}\right),
$$

where $a_{0}$ is the wire radius and $\rho$ is the maximum radius of the cone at the screen. For small wires, $a_{0}$ $\ll \rho$, and Eq. (7) is equivalent to Eqs. (6).

As the wire is illuminated with an intense beam, several cones are generated, one for each location $z$ illuminated by the beam $[z$ is the distance from the wire to the screen, as defined in Fig. 2(b)]. As a consequence, the circle of light at the screen has a width that depends on the beam width. If the beam is wide enough with respect to the wavelength (to 
avoid diffraction effects), and when geometrical optics is valid ( $\lambda \ll a_{0}$ ), there is a correspondence between the wire's location $z$ and the radius of each circumference at the screen $\rho$. When the incident rays are parallel, this correspondence is

$$
z=\frac{\rho}{\tan \theta_{2}} .
$$

When the rays are divergent (as in our experimental setup as a result of the cylindrical lens; see Subsection 4.A below), each ray has a different incidence angle

$$
\tan \theta_{1}=\frac{h}{L-z},
$$

where $L$ is the distance between the lens and the screen and $h$ is the distance between the lens and the wire axis. In this case the relationship between angle $\rho$ and the location at the wire $z$ is

$$
z=\frac{\rho L}{\rho+h} .
$$

Another point to be considered is the intensity at the cone. If the wire surface is assumed to be an ideal reflector (reflectance factor, $R=1$ ), the intensity at an angle $\phi_{2}$ is proportional to the effective area, that is, $I\left(\theta_{2}, \phi_{2}\right) \propto\left|\mathbf{k}_{1} \cdot \hat{n}\right|$. When the incident beam is a plane wave, the intensity at the cone is

$$
I_{\text {theor }}\left(\theta_{2}, \phi_{2}\right)=I_{0} \delta\left(\theta_{2}-\theta_{1}\right)\left|\cos \left(\phi_{2} / 2\right)\right|,
$$

where $I_{0}$ is the intensity at $\phi_{2}=0$ and $\delta\left(\theta_{2}-\theta_{1}\right)$ is the Dirac delta function.

\section{Detection of Surface Defects}

The intensity distribution at the cone can be used to characterize the surface of a thin metallic wire. When the wire shows surface defects, the correspondence between the cone of light and the wire surface is no longer valid for the places where the defects occur, because rays at these locations are sent to different directions from those for a wire without defects. Then it is possible to associate, at a given location, the decrease of intensity with respect to that obtained by Eq. (11) with a defect at this location. In Fig. 3 the intensity ring obtained at the screen is shown for a wire that has scratches. To detect the defects we define a function $I_{D}$, which give us the locations of the defects at the wire:

$$
I_{D}\left(\phi_{1}, z\right)=I\left(\phi_{1}, z\right)-I_{F}\left(\phi_{1}, z\right) .
$$

$I\left(\phi_{1}, z\right)$ is simply the intensity at the ring when the coordinate system has been changed in accordance with Eqs. (6), (8), and (11) and $I_{F}\left(\phi_{1}, z\right)$ is the theoretical intensity distribution for a wire without defects. For the ideal case of plane-wave illumination, $I_{F}\left(\phi_{1}, z\right)$ is given by Eq. (11). However, experimentally it is not possible to control all the parameters involved (beam widths, misalignments between wire and beam, reflectance of the surface, etc.). For this

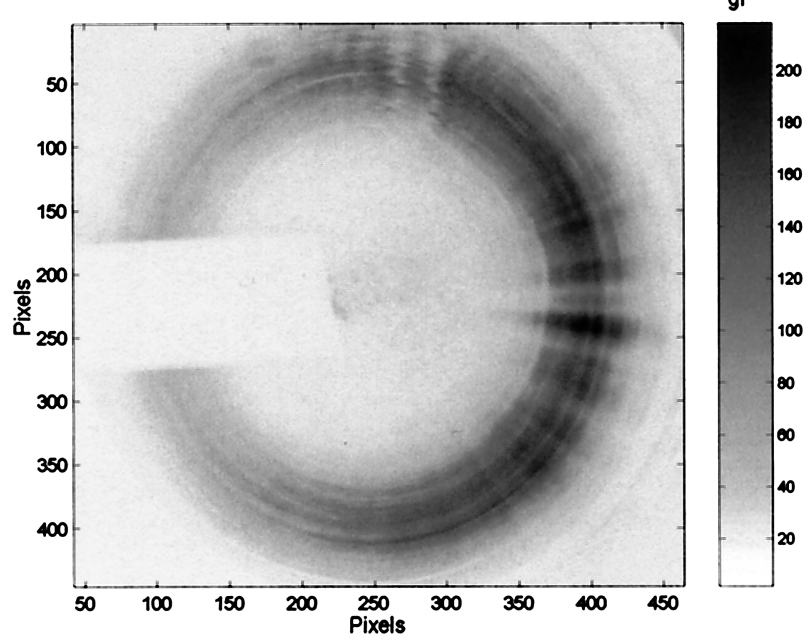

Fig. 3. (a) Image of the ring detected by the CCD camera for a steel wire (diameter, $300 \pm 1 \mu \mathrm{m}$ ) with scratches.

reason we obtain $I_{F}\left(\phi_{1}, z\right)$ by filtering $I\left(\phi_{1}, z\right)$ with a low-pass filter to eliminate short-range intensity fluctuations. The defects are placed at the locations where $I\left(\phi_{1}, z\right)<I_{F}\left(\phi_{1}, z\right)$. Besides, to eliminate noise and small defects whose presence it is not necessary to detect, we have defined a threshold level $I_{T}$ in such a way that the defects are detected if

$$
I_{D}\left(\phi_{1}, z\right)<I_{T} .
$$

A correct value for $I_{T}$ is important for the defects to be discriminated properly. We can obtain this value by introducing a high-quality wire into our system and making $I_{T}$ the value at which no defects are detected by $I_{D}\left(\phi_{1}, z\right)$.

Inasmuch as the cylindrical geometry of the wire acts as an image amplifier in the transverse direction, the defects that are most easily detected are longitudinal ones, such as scratches and protuberances. However, other kinds of defect, such as holes and flakes, can also be detected. In the case of rough wires, for which the density of defects is so high that statistical methods need to be applied, the proposed method is still valid, but it is necessary to remove the cylindrical lens. ${ }^{11}$ One obtains the roughness pa-

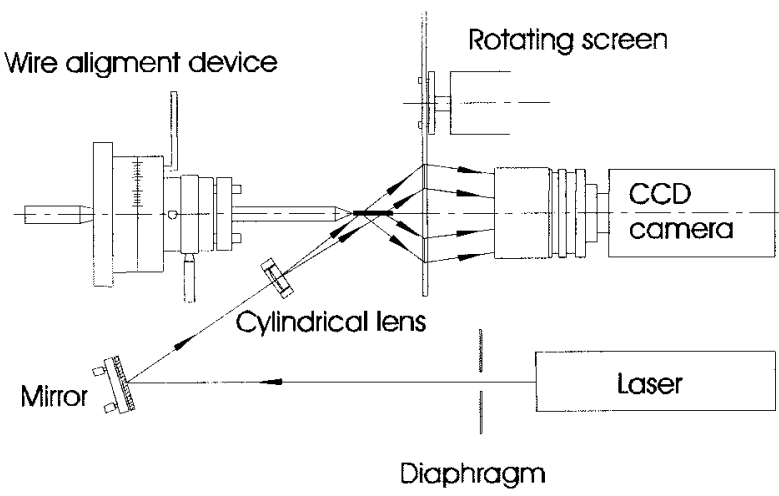

Fig. 4. Schematic diagram of the experimental setup. 
rameters by measuring the width of the circumference.

The defects are best detected at $\phi_{1}=0$, because the intensity at this angle is a maximum. As we depart from this angle, the defects are poorly detected, and they cannot be detected at all at $\phi_{1}= \pm \pi / 2$. At that point, to characterize the wire completely we require at least two incident beams $180^{\circ}$ apart that generate two cones. Each cone gives us the information for half of a wire.

Often the interest is not in actually obtaining the location of the defects but in measuring the surface quality of the wires. A procedure for doing this is to measure the relative area of the defects. Accordingly, we propose the following quality parameter:

$$
\Gamma(z)=\frac{1}{\pi} \int_{\Omega} D\left(z, \phi_{1}\right) \mathrm{d} \phi_{1},
$$

where

$$
D\left(\phi_{1}, z\right)= \begin{cases}1 & I_{D}\left(\phi_{1}, z\right)<I_{T} \\ 0 & I_{D}\left(\phi_{1}, z\right)>I_{T}\end{cases}
$$

(a)

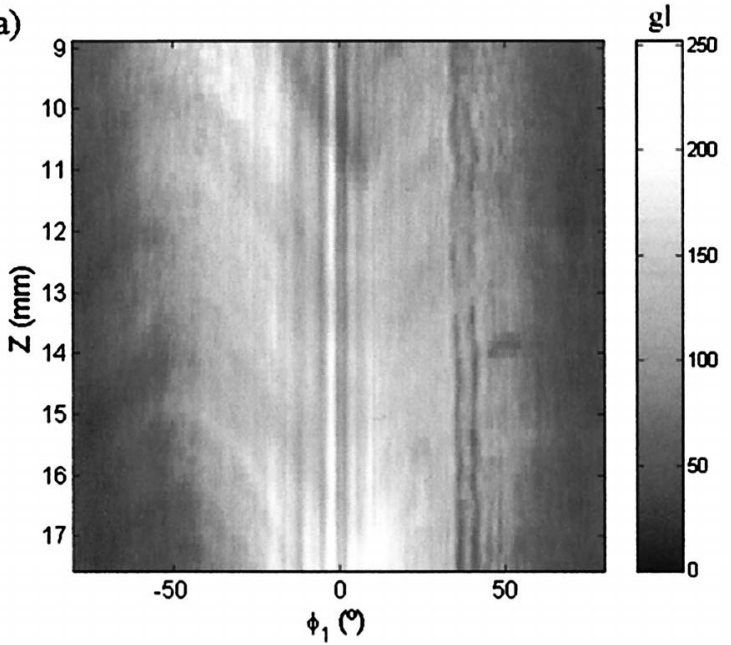

(b)

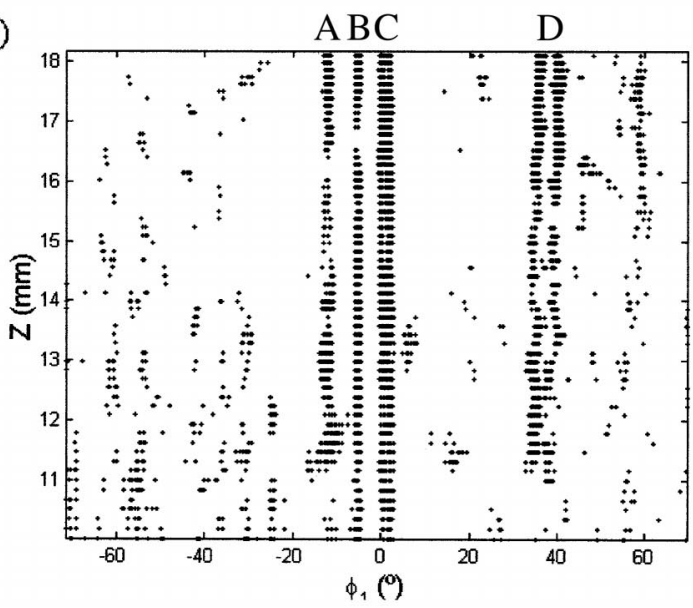

Fig. 5. (a) Reconstruction, by means of geometrical transformation, of the wire shown in Fig. 3. This reconstruction is an intensity image of the wire. (b) Defects detected by $I_{D}\left(\phi_{1}, z\right)$ when $I_{T}=$ $-10 \mathrm{gl}$.

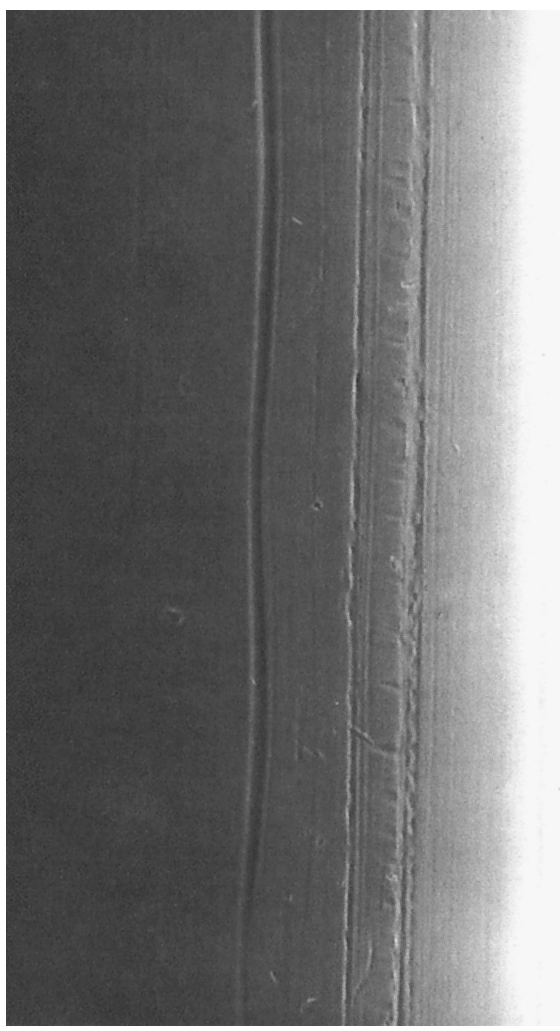

Fig. 6. SEM image of the steel wire with defects of Fig. 3. We can see three scratches at the center of the image and another scratch at the right in the image.

and $\Omega$ are the angles $\phi_{1}$ to be considered in integration. Equation (14) is a measurement of the percentage of defects over the wire surface. Because the area that contributes diffraction to the intensity is removed by a mask, as explained in Subsection 4.A, the integration angles are $\Omega=\left(-\phi_{0}, \phi_{0}\right)$, where $\phi_{0}$ is the angle of the mask edge.

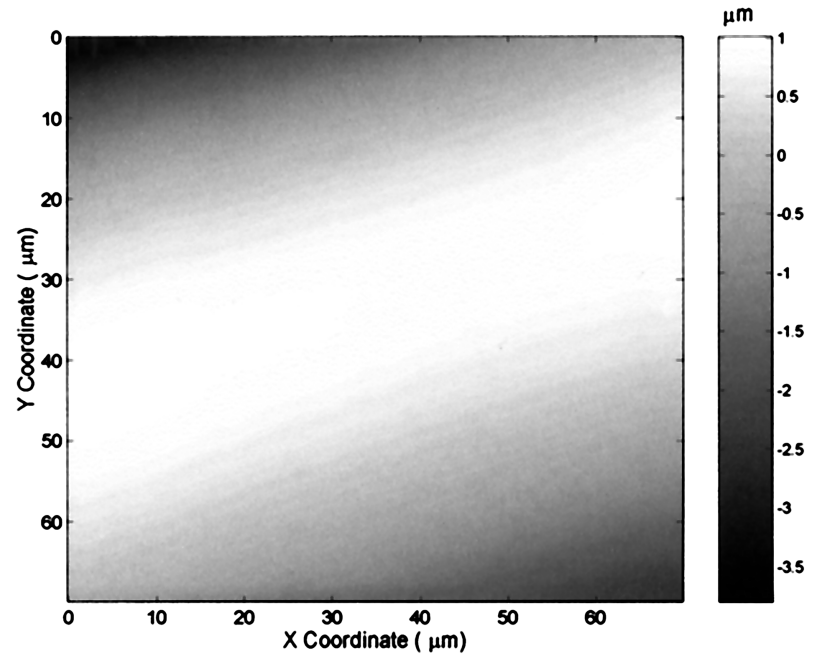

Fig. 7. AFM image of a steel wire (diameter, $399 \pm 1 \mu \mathrm{m}$ ) with a high-quality surface. As we can observe, no defects are detected by the AFM. The resolution of the AFM is $\pm 6 \mathrm{~nm}$ in height. 
(a)

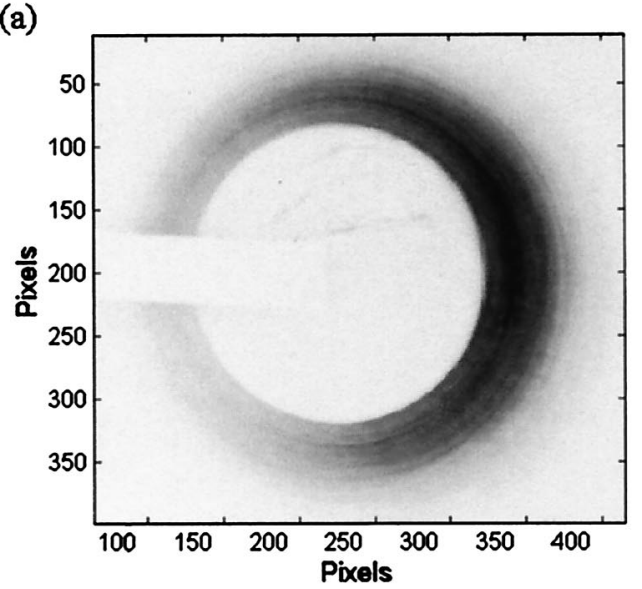

(b)

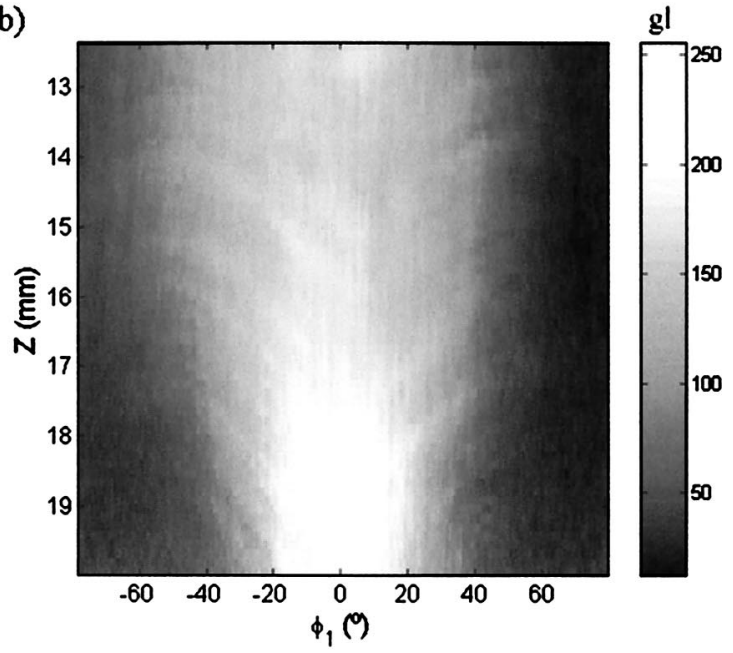

(c)

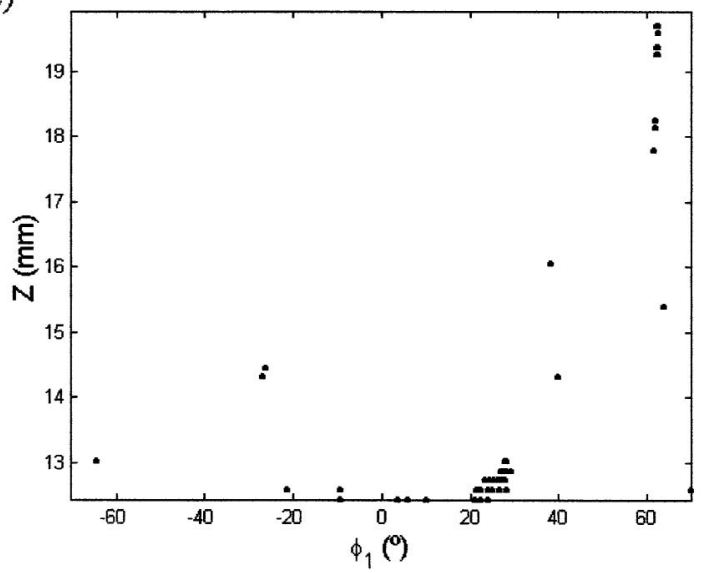

Fig. 8. (a) Image of the ring detected by the CCD camera for the steel wire of Fig. 7. (b) Image of the geometrical reconstruction. (c) Defects detected by $I_{D}\left(\phi_{1}, z\right)$ when $I_{T}=-10 \mathrm{gl}$.

\section{Experimental Method}

\section{A. Setup}

The experimental setup is represented in Fig. 4. A $\mathrm{He}-\mathrm{Ne}$ laser beam $(\lambda=632.8 \mathrm{~nm})$ is horizontally expanded by a cylindrical lens to increase the illumi-
Table 1. Width and Distance between Scratches (in micrometers) for the Wire with Surface Defects shown in Fig. $5^{a}$

\begin{tabular}{cccccccc}
\hline $\begin{array}{c}\text { Measurement } \\
\text { Method }\end{array}$ & $A$ & $d(A, B)$ & $B$ & $d(B, C)$ & $C$ & $d(C, D)$ & $D$ \\
\hline Cone & 3.9 & 14.4 & 2.6 & 11.7 & 6.5 & 80 & 7.0 \\
SEM & 3.0 & 14.9 & 2.0 & 9.4 & 4.2 & 66 & 6.2 \\
\hline
\end{tabular}

${ }^{a}$ These parameters were measured by SEM and by our cone technique. $A$ is the width of a scratch, and $d(A, B)$ means the distance between scratches A and B.

nated area on the wire. The distance from the lens to the wire is $h=5 \mathrm{~cm}$, and the distance from the lens to the screen is $L=12 \mathrm{~cm}$. The wire is not uniformly illuminated by this beam; its intensity distribution is

$$
I(x, y)=I_{L} \exp \left[-\left(x / L_{x}\right)^{2}-\left(y / L_{y}\right)^{2}\right],
$$

where $L_{x} \approx 6 \mathrm{~mm}$ and $L_{y} \approx 1 \mathrm{~mm}$ are the horizontal and vertical half-widths of the beam, respectively, at $1 / e$. The nonuniformity of the illumination is not important because it is eliminated by the imageprocessing algorithm. The beam impinges upon the wire, and the output rays, which form a cone as we have pointed out, reach a rotating screen that is used to prevent speckle produced by the laser beam. The image on the rotating screen is recorded by a two-dimensional CCD camera [512 $\times 512$ pixels, 256 gray levels (gl)]. A mask consisting of a slit in front of the screen is used to prevent saturation at the diffraction maximum. Because the mask produces an abrupt intensity variation at the cone, it affects the detection algorithm. At the mask edges the algorithm detects what appears to it as a defect. However, as the angles of the mask edges are well known, these spurious defects can easily be eliminated from calculations.

To obtain the correspondnce between size and number of pixels, we place a $3 \mathrm{~cm} \times 3 \mathrm{~cm}$ square at the screen and measure the number of dark pixels in the horizontal and vertical directions. In this way

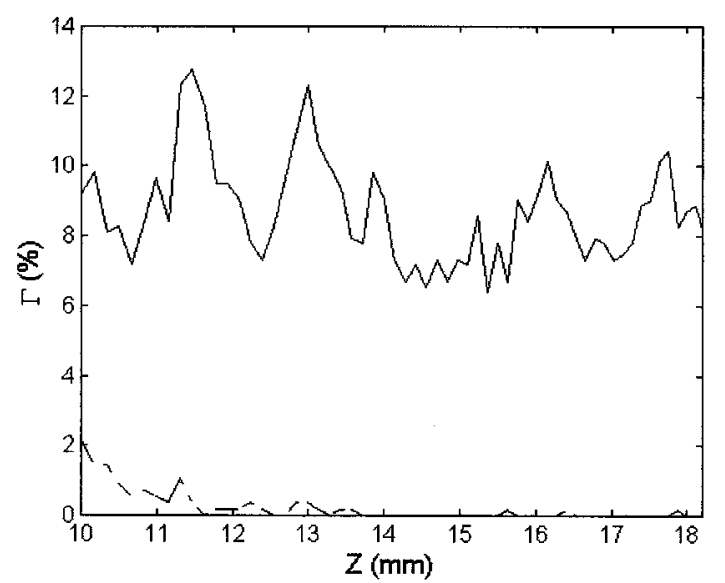

Fig. 9. $\Gamma(z)$ parameter (solid curve) for the wire with defects of Fig. 3 and (dashed curve) for the high-quality surface wire of Fig. 7. 
we can measure the horizontal and vertical amplification of the CCD camera.

\section{B. Results}

With our setup we measured several wires with surface defects. In all these measurements we found scratches, protuberances, or both. Our measurements by SEM and AFM also indicate that these are the most common defects on a wire surface. ${ }^{5}$ For example, Fig. 3 shows the intensity ring for a steel wire with scratches (diameter, $300 \pm 1 \mu \mathrm{m}$ ). By means of Eqs. (6), (8), and (10) we obtained the reconstruction $I\left(\phi_{1}, z\right)$ of the wire surface from the ring $I\left(\theta_{2}, \phi_{2}\right)$, as one can observe in Fig. 5(a). To detect the defects by using an automatic algorithm we filtered the reconstructed image $I\left(\phi_{1}, z\right)$, as is explained in Section 3. The locations that fulfill Eq. (13), $I_{T}=$ $-10 \mathrm{gl}$, are the locations where the defects are placed, and they are represented in Fig. 5(b). This image can be compared with a SEM image of the wire surface (Fig. 6). In both images we can see three long scratches $(\mathrm{A}, \mathrm{B}, \mathrm{C})$ at the center and another big scratch at the right (D). Both images are intensity images, which give us information about the surface, but they are not topographical images such as those obtained by AFM, and we cannot measure the depths of the defects. However, our system can measure approximately $1-2 \mathrm{~cm}$ of the wire in only one image, whereas with SEM or AFM the measurement range is $\sim 1 \mathrm{~mm}$.

To determine threshold level $I_{T}$ we previously measured a steel wire (diameter, $399 \pm 1 \mu \mathrm{m}$ ) whose biggest defect was $\sim 20 \mathrm{~nm}$ deep, as shown in the AFM image of the wire (Fig. 7). The intensity ring obtained for this wire is quite uniform [Fig. $8(\mathrm{a})]$, and the reconstructed intensity image of the wire $I\left(\phi_{1}, z\right)$ is also homogeneous [Fig. 8(b)]. Noise is eliminated when $I_{T}=-10 \mathrm{gl}$, as we can see from Fig. 8(c).

We also measured the widths of the scratches and the distance between scratches for Fig. 5(b) and compared the results with those obtained by SEM (Fig. 6). Because the SEM images are projections we need to perform some simple trigonometric calculations to obtain true distances from projected distances. The results are shown in Table 1.

Finally, the $\Gamma(z)$ parameter was measured for several wires with surface defects and showed a correlation between $\Gamma(z)$ and the quantity of defects on the wire surface. In Fig. 9 we show $\Gamma(z)$ for the wire with surface defects shown in Fig. 3 and for the wire without defects shown in Fig. 7. The mean values for $\Gamma(z)$ are $0.2 \%$ for the high-quality wire and $8.7 \%$ for the wire with defects.

\section{Conclusions}

We have proposed an automatic optical technique to detect surface defects on thin metallic wires that can be implemented in on-line automatic systems for wire inspection. The defects are detected by measurement of the intensity variations at the ring that is produced when the wire is illuminated with a laser beam at oblique incidence. The defects that are most easily detected are scratches and protuberances along the wire, although other defects such as holes and noses can also be detected. We also propose a quality parameter that provides us a quantitative surface classification for the wires. The results predicted by this technique have been compared with our measurements obtained by scanning-electron microscopy and atomic-force microscopy.

The authors are grateful to A. Gonzalez-Cano for his suggestions and discussions in the development of this research. The authors also express their gratitude to the European Union (EU) for the financial support of this research within the framework of EU research program SMT4, Standards Measurement and Testing; project SMT4-CT97-2184, DEFCYL: Detection of Defects in Cylindrical Surfaces, and Comisión Interministerial de Ciencia y Tecnología of Spain project TAP-98-1358-CE.

\section{References}

1. T. K. Millard and T. A. Herchenreder, "Automatic diameter measurement: state of the art," Wire J. Intern. 24(12), 61-69 (1991).

2. J. F. Fardeau, "New laser sensor for wire diameter measurement," Wire J. Intern. 22(1), 42-51 (1989).

3. I. Serroukh, E. Bernabeu, and L. M. Sanchez-Brea, "A geometrical model for wire optical diffraction selected by experimental statistical analysis," Opt. Eng. 34, 1319-1325 (1999).

4. G. W. Rowe, An Introduction to the Principles of Metalworking (Edward Arnold, London, 1965).

5. L. M. Sanchez-Brea, J. A. Gomez-Pedrero, and E. Bernabeu, "Measurement of surface defects on thin steel wires by atomic force microscopy," Appl. Surf. Sci. 150, 125-130 (1999).

6. D. J. Whitehouse, "Surface metrology," Meas. Sci. Technol. 8, 955-972 (1997).

7. C. López, A. F. Doval, B. V. Dorrío, J. Blanco-García, J. Bugarín, J. M. Alén, A. Fernández, J. L. Fernández, M. Pérez-Amor, and B. G. Tejedor, "Fibre optic reflectometric technique for the automatic detection and measurement of surface cracks," Meas. Sci. Technol. 9, 1413-1431 (1998).

8. S. Gómez, K. Hale, J. Burrows, and B. Griffiths, "Measurements of surface defects on optical components," Meas. Sci. Technol. 9, 607-616 (1998).

9. K. I. Jolic, C. R. Nagarajah, and W. Thompson, "Non-contact, optically based measurement of surface roughness of ceramics," Meas. Sci. Technol. 5, 671-684 (1994).

10. L. M. Sanchez-Brea, P. Siegmann, E. Bernabeu, F. PérezQuintian, M. A. Rebollo, and C. A. Raffo, "Medición de la Rugosidad y localización de fallas en hilos metálicos por métodos ópticos," presented at the 83rd Reunión Nacional de Física, La Plata, Argentina, 21-25 September 1998.

11. F. Perez-Quintian, M. A. Rebollo, N. G. Gaggioli, and C. A. Raffo, "Optical methods for on line surface wire testing," presented at the Seventh European Conference on Nondestructive Testing, Copenhagen, Denmark, 26-29 May 1998.

12. S. A. Stefani, C. R. Nagarajah, and R. Willgoss, "A surface inspection technique for continuously extruded cylindrical products," Meas. Sci. Technol. 10, N21-N25 (1999).

13. T. K. Millard, "Computer simulation of defect detection," Wire Ind. 58, 141-145 (1991). 
14. C. Babu Rao, A. V. Ananthalakshmi, and R. Kesavamoorthy, "Laser scattering from the surface of thin wires at oblique illumination," in Optics and Optoelectronics Theory, Devices and Applications, O. Nijhawan, A. K. Gupta, A. K. Musla, and K. Singh, eds. (Narosa, House, New Delhi, 1999), pp. 298-301.

15. C. Babu Rao, A. V. Ananthalakshmi, and R. Kesavamoorthy, "Localization of surface roughness of thin wires using laser scattering," presented at the Fourteenth World Conference on Nondestructive Testing, New Delhi, 8-13 December 1996.

16. J. B. Keller, "Geometrical theory of diffraction," J. Opt. Soc. Am. 52, 116-130 (1962).

17. H. Hönl, A. W. Maue, and K. Westpfahl, "cap. B-II, Beugung un konvexen Körpern ohne Kanten," in Handbuch der Physik, S. Flügge, ed. (Springer-Verlag, Berlin, 1961), Vol. XXV, p. 1. 